\begin{tabular}{|c|c|c|}
\hline Beitr. Ent. & Berlin & ISSN 0005-805X \\
\hline $\mathbf{5 0}(2000) 1$ & S. $91-101$ & 11.04 .2000 \\
\hline
\end{tabular}

\title{
Contribution to the knowledge of the Jewel Beetles of Albania
}

\section{(Coleoptera: Buprestidae)}

With 2 figures and 1 table

\section{VLADIMIR P. SAKALIAN}

\section{Summary}

A total number of 48 species and subspecies belonging to 18 genera and 8 subfamilies of Buprestidae are reported from Albania. 15 taxa marked by asterisk in the text are new for the country's fauna. Including the new records, the number of jewel beetles known from the Albanian territory has increased to 111 . The new localities for the reported buprestids are listed. A zoogeographical analysis based on all known Albanian jewel beetles is presented. The dominance of Mediterranean elements indicates a high impact of the Mediterranean fauna on the Albanian buprestid communities.

\section{Key words}

Coleoptera, Buprestidae, Albania, Mediterranean, Faunistics, Zoogeography

\section{Zusammenfassung}

Es wird über insgesamt 48 Arten und Unterarten von Albanien, die zu 18 Gattungen und 8 Unterfamilien der Familie Buprestidae gehören, berichtet. Die 15 für die Fauna des Landes neuen Taxa sind mit einem Sternchen markiert. Mit den gesammelten neuen Arten erhöht sich die Zahl der Buprestiden, die aus Albanien bekannt sind, auf 111. Angefïgt ist eine zoogeographische Analyse aller bekannten albanischen Buprestiden. Das Überwiegen von mediterranen Arten zeigt den starken Einfluß der Mittelmeerfauna auf die Zusammensetzung der Buprestidenfauna Albaniens.

\section{Acknowledgments}

I wish to thank Dr S. Bíly (Prague) and Dr M. KALASHIAN (Erevan) for the determination and confirmation of some species. I am obliged to my Bulgarian colleagues S. ABADJEV, Dr P. BERON, G. Blagoev, B. Guéorguiev, Dr J. Kolarov, M. Langourov, Dr L. PeneV and B. Petrov, for accepting the buprestid species for investigation. Special thanks to Dr K. MISJA and Dr O. MERKL for giving me the possibility to work with the collections of the Museums in Tirana and Budapest.

\section{Introduction}

After the summarizing paper of MÜHLE (1980) and its supplement (MÜHLE, 1984) so far there have been no other publications on the Albanian buprestids fauna. VOLKOVITSH (1989) in his work for buprestids from Eastern Mediterranea reported another one species (Acmaeoderella jonica).

The main goal of this paper is to present new faunistical and zoogeographical information about the Buprestidae family from this very interesting area of the Balkan Peninsula. 


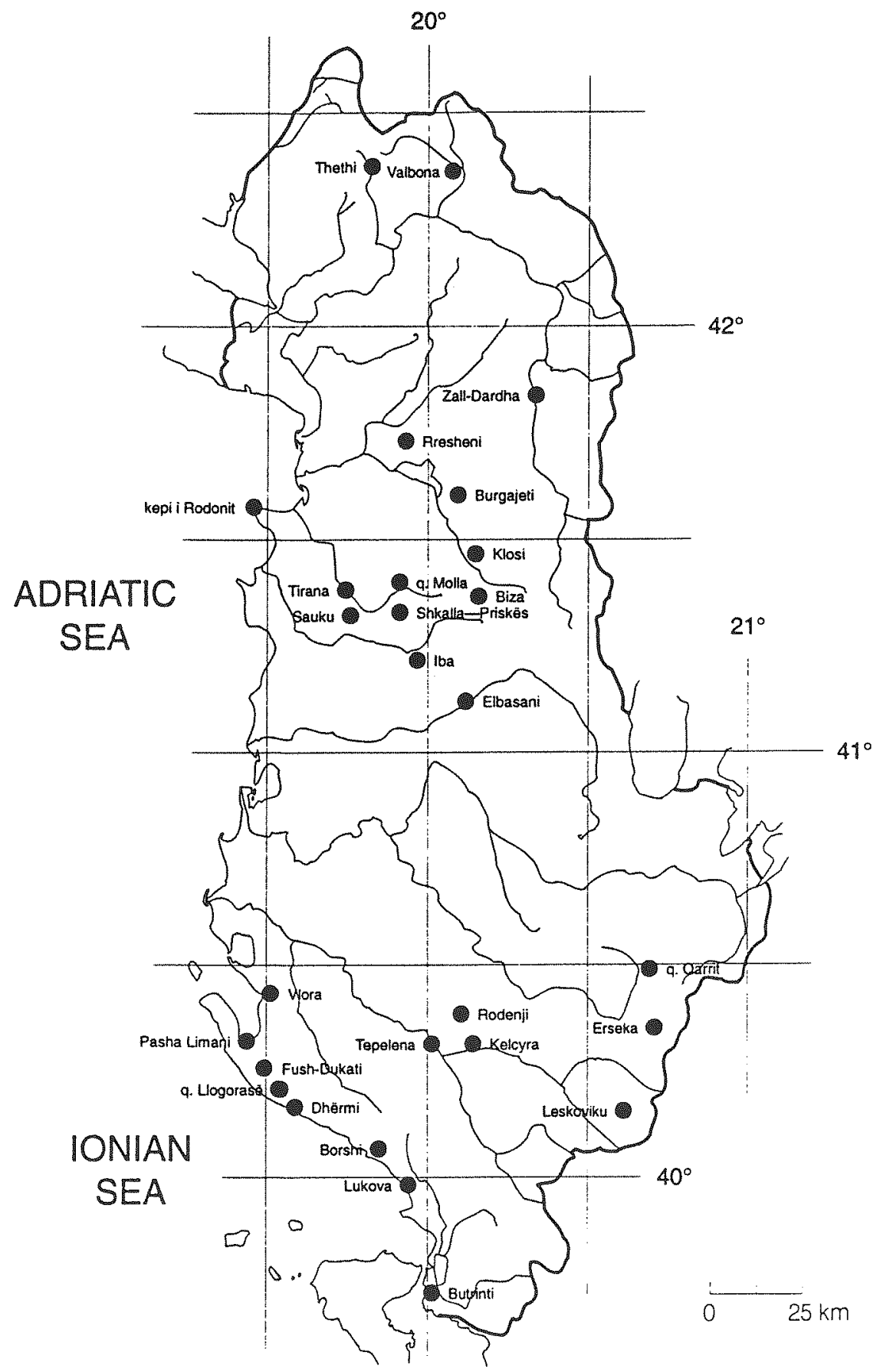

Fig. 1. Map of Albania showing the localities. 


\section{Material and Methods}

The greater part of studied material was collected by the author in 1994 and 1995. Traditional faunistical methods for buprestids catching were used: sweeping grass vegetation, beating of the trees and bushes branches, decortication of the trees and bushes etc. The collected species are kept in the Institute of Zoology Scientific Funds (IZ). Material and data from the following collections have been used: Museums of Natural History in Tirana (Muzeu Shkencave Natyrore - MSN) and in Budapest (Termeszettudomanyi Muzeum, Budapest - TMB). The localities included in this paper are presented in Fig. 1.

The zoogeographical classification of the jewel beetles is based mainly on the data of their recent spreading. When the species are polytypic and subspecies known for Albania are morphologically distinct or geographically separated, then the information of the distribution of concrete subspecies is used.

The list of the established species and subspecies comprises the following data: the valid taxa name; the information for provinces, concrete locality names (Fig. 1), date of catching, number of found specimens, the name of the collector or determinator (when the material was not collected or determined by the author), the name of collections where the taxa are kept and the plants where some species were found.

The new taxa for the Albanian fauna are marked by asterisk in the list.

\section{Faunistical Results}

The established buprestids taxa are as follow:

JULODINAE

*Julodis ehrenbergi LAPORTE, 1835

Albania, 2 ex. (coll. FUss in TMB).

POLYCESTINAE

Ptosima flavoguttata (ILLIGER, 1803)

Prov. Tirana: Shkalla-Priskès, 29.06.1958, 1 ex, leg. X. MURRAJ (coll. MSN).

\section{ACMAEODERINAE}

* Acmaeodera brevipes brevipes KIESENWETTER, 1858

Prov. Saranda: Lukova, 5.06.1995, 1 ex (coll. IZ).

Acmaeodera bipunctata bipunctata (OLIVIER, 1790)

Prov. Korca: q. Qarrit, 1.06.1994, 1 ex; Prov. Kolonja: Leskoviku, 1.06.1994, 1 ex, 2.06.1995, 7 ex (coll. IZ).

Acmaeodera crinita crinita SPINOLA, 1838

Prov. Saranda: Lukova, 5.06.1995, 3 ex; Prov. Vlora: Vlora, 7.06.1995, 1 ex (coll.

IZ).

*Acmaeodera ottomana (FRIVALDSZKY, 1837)

Prov. Saranda: Butrinti, 4.06.1995, 2 ex, Lukova, 5.06.1995, 2 ex (coll. IZ).

*Acmaeoderella adspersula adspersula (ILLIGER, 1803)

Prov. Saranda: Butrinti, 4.06.1995, 1 ex, Lukova, 5.06.1995, 1 ex (coll. IZ). Acmaeoderella flavofasciata flavofasciata (PILLER \& MITTERPACHER, 1783)

Prov. Permeti: Rodenji, 22.07.1962, 1 ex, leg. X. MURRAJ (coll. MSN).

*Acmaeoderella mimonti (BoIELDIEU, 1865)

Prov. Saranda: Lukova, 5.06.1995, 1 ex (coll. IZ). 


\section{CHALCOPHORINAE}

Chalcophora mariana (LINNAEUS, 1758)

Prov. Mati: Burgajeti, 9.08.1959, 1 ex, leg. X. MURRAJ (coll. MSN); Prov Kolonja: Leskoviku, 1.06.1994, 14 ex, coll. on the trunks of Pinus sp. (coll. IZ) . Chalcophorella stigmatica (SCHÖNHERR, 1817)

Prov. Vlora: Fush-Dukati, 7.05.1958, 1 ex, 8.06.1958, 1 ex, 6.07.1959, $1 \mathrm{ex}$ leg. X. MurraJ (coll. MSN); Prov. Saranda: Butrinti, 24.06.1995, 1 ex, leg. S. ABADJIEV (coll. IZ).

Chalcophorella fabricii (Rossi, 1794)

Prov. Vlora: Pasha Limani, 05.1908, 1 ex (coll. TMB).

Capnodis tenebricosa (OLIVIER, 1790)

Prov. Tirana: Iba, 4.07.1963, 1 ex, leg X. MurRaJ (coll. MSN); Prov. Vlora: Dhèrmi, 26.06.1995, 1 ex, leg. S. ABADJIEv; Prov. Saranda: Lukova, 3-4.06.1994, 2 ex, 3.06.1995, 1 ex (coll. IZ).

Capnodis tenebrionis (LINNAEUS, 1761)

Prov. Tirana: Sauku, 4.05.1959, 1 ex, leg. X. MurRaJ (coll. MSN); Prov. Saranda: Lukova, 5.06.1995, 1 ex (coll. IZ).

Capnodis cariosa (PALlas, 1776)

Prov. Permeti: Kelcyra, 16-20.08.1962, 1 ex, leg. X. MurraJ (coll. MSN); Prov. Kolonja: Leskoviku, 1.06.1994, 1 ex; Prov. Saranda: Lukova, 3.06.1994, 3 ex on Phylirea media; Prov. Vlora: Vlora, 5.06.1994, 1 ex, Dhèrmi, 26.06.1996, 1 ex (coll. IZ).

Capnodis porosa (KLUG, 1829)

Prov. Kolonja: Leskoviku, 1.06.1994, 1 ex (coll. IZ).

Aurigena lugubris lugubris (FABRICIUS, 1777)

Prov. Didra: Zall-Dardha, 23.05.1958, 1 ex; Prov: Permeti: Kelcyra, 16-20.08. 1962, 1 ex; Prov. Tirana: Tirana, 4.10.1966, 1 ex, leg. X. MURRAJ (coll. MSN); Prov. Saranda: Lukova, 3-4.06.1994, G. BLAGoEv; Prov. Vlora: Dhèrmi, 26.06. 1995, 2 ex, leg. S. ABADJIEV (coll. IZ).

\section{SPHENOPTERINAE}

* Sphenoptera lapidaria BRULLÉ, 1832

Prov. Tepelena: Tepelena, 3.06.1995, 1 ex on Platanus orientalis, det. M. KALASHIN (coll. IZ).

* Sphenoptera tappesi MARSEUL, 1865

Prov. Saranda: Lukova, 5.06.1995, 1 ex on Olea sp. (coll. IZ).

\section{BUPRESTINAE}

Dicerca aenea (LINNAEUS, 1761)

Prov. Tepelena: Tepelena, 2.06.1994, 1 ex (coll. IZ).

Dicerca berolinensis (HERBST, 1779)

Prov. Tirana: Biza, 9-16.07.1961, 4 ex leg. X. MurraJ (coll. MSN); Prov. Mirdita: Rresheni, 11.06.1993, 1 ex, leg. P. BERON and B. PETROV (coll. IZ).

Scintillatrix mirifica MULSANT, 1855

Prov. Elbasani: Elbasani, 20.06.1994, 1 ex, leg. J. KolAROV and M. LANGOUROV (coll. IZ).

* Eurythyrea austriaca (LINNAEUS, 1767)

Prov, Tropoja: Valbona, 28.07.1959, 1 ex, leg. X. MURRAJ (coll. MSN). 
Eurythyrea aurata (PALLAS, 1776)

Prov. Tirana: Sauku, 10-20.06.1961, 1 ex, leg. X. MURRAJ (coll. MSN).

Buprestis haemorrhoidalis haemorrhoidalis HERBST, 1780

Prov. Kolonja: Leskoviku, 9.07.1959, 1 ex, leg. X. MurRAJ (coll. MSN).

Buprestis novemmaculata novemmaculata LINNAEUS, 1767

Prov. Mati: Burgajeti, 9.08.1959, 1 ex, leg. X. MURRAJ (coll. MSN).

* Buprestis novemmaculata gravida ABEILLE DE PERRIN, 1904

Prov. Didra: Zall-Dardha, 25.05.1958, 1 ex, leg. X. MURRAJ (coll. MSN).

Buprestis splendens FABRICIUS, 1774

Prov. Shkodra: Thethi, 21.07.1959, 1 ex, leg.X. MuRRAJ (coll. MSN).

Anthaxia hungarica hungarica (SCOPOLI, 1772)

Prov. Kolonja: Leskoviku, 2.06.1995, 1 ex; Prov. Saranda: Butrinti, 4.06.1995, 2

ex (coll. IZ).

* Anthaxia diadema diadema (FisCHER, 1823)

Prov. Permeti: Rodenji, 2-22.07.1962, 1 ex, leg. X. MURRAJ (coll. MSN).

Anthaxia millefolii millefolii (FABRICIUS, 1801)

Prov. Saranda: Butrinti, 3.06.1994, 4 ex, 4.06.1995, 14 ex, Lukova 4.06.1994,

2 ex, 5.06.1995, 2 ex; Prov. Mati: Klosi, 8.06.1995 1 ex (coll. IZ).

Anthaxia umbellatarum umbellatarum (FABRICIUS, 1787)

Prov. Saranda: Lukova, 3-4.06.1994, 7 ex, 5.06.1995, 1 ex (coll. IZ).

*Anthaxia scutellaris scutellaris GENE, 1839

Prov. Saranda: Lukova, 5.06.1995, 1 ex, det. S. BíLÝ (coll. IZ).

Anthaxia cichorii cichorii (OLIVIER, 1780)

Prov. Tirana: q. Molla, 17.06.1960, 1 ex; Prov. Tepelena: Tepelena, 30.05.1961,

1 ex; Prov. Tirana: Sauku, 16.05.1962, 1 ex, leg. X. MurraJ (coll. MSN); Prov.

Durres: kepi i Rodonit, 21.06.1994, 1 ex, leg. B. GuÉoRGUIEv; Prov. Saranda:

Lukova, 4-5.06.1994, 3 ex, Butrinti, 4.06.1995, 5 ex (coll. IZ).

Anthaxia istriana ROSENHAUER, 1847

Prov. Kolonja: Erseka, 12.05.1995, 1 ex, L. PENEv, Leskoviku, 2.06.1995, 2 ex;

Prov. Korca: q. Qarrit, 1.06.1995, 2 ex (coll. IZ).

*Anthaxia godeti LAPORTE \& GORY, 1839

Prov. Korca: q. Qarrit, 1.06.1994, 5 ex; Prov. Kolonja: Leskoviku, 2.06.1995, 1 ex (coll. IZ).

* Anthaxia brevis brevis LAPORTE \& GORY, 1839

Prov. Saranda: Butrinti, 3.06.1994, 2 ex (coll. IZ).

CHRYSOBOTHRINAE

Chrysobothris affinis (FABRICIUS, 1794)

Prov. Tirana: Biza, 9-16.07.1961, 1 ex, leg. X. MURRAJ (coll. MSN).

\section{AGRILINAE}

Coroebus rubi (LINNAEUs, 1767)

Prov. Tirana: Tirana, 1.06.1958, 1 ex, 10.05.1961, 1 ex, Iba 4.07.1963, 1 ex; Prov. Permeti: Rodenji, 26-28.07.1962, 1 ex, leg. X. MURRAJ (coll. MSN); Prov. Saranda: Butrinti, 4.06.1995, 1 ex (coll. IZ).

Coroebus elatus (FABRICIUS, 1787)

Prov. Permeti: Rodenji, 2-22.07.1962, 1 ex, leg. X. MurRaJ (coll. MSN); Prov.

Korca: q. Qarrit, 1.06.1994, 1 ex; Prov. Vlora: q. Llogorasè, 5.06.1994, 1 ex; Prov.

Kolonja: Leskoviku, 2.06.1995, 1 ex (coll. IZ). 
Nalanda fulgidicollis (LUCAS, 1849)

Prov. Korca: q. Qarrit, 1.06.1994, 6 ex on Quercus sp.; Prov. Saranda: Lukova, 3-

4.06.1994, 1 ex on Quercus coccifera (coll. IZ).

*Agrilus convexicollis convexicollis REDTENBACHER, 1849

Prov. Saranda: Butrinti, 3.06.1994, 1 ex, 4.06.1995, 1 ex on Olea sp. (coll. IZ).

* Agrilus dualis dualis ALEXEEv \& BíLÝ, 1980

Prov. Saranda: Butrinti, 4.06.1995, 3 ex on Quercus coccifera (coll. IZ).

Agrilus laticornis (ILLIGER, 1803)

Prov. Korca: q. Qarrit, 1.06.1994, 1 ex on Quercus sp. (coll. IZ).

Agrilus obscuricollis KIESENWETTER, 1857

Prov. Korca: q. Qarrit, 1.06.1994, 1 ex on Quercus sp. (coll. IZ).

Agrilus angustulus (ILLIGER, 1803)

Prov. Korca: q. Qarrit, 1.06.1995, 1 ex on Quercus sp.; Prov. Saranda: Butrinti,

4.06.1995, 4 ex on Quercus coccifera (coll. IZ).

Agrilus auricollis KIESENWETTER, 1857

Prov. Saranda: Borshi, 13-28.05.1961, 1 ex, leg. X. MurRAJ (coll. MSN).

Agrilus roscidus KIESENWETTER, 1857

Prov. Saranda: Lukova, 3-4.06.1994, 1 ex, 5.06.1995, 6 ex on Olea sp.; Prov.

Kolonja: Leskoviku, 2.06.1995, 1 ex on Arbutus sp. (coll. IZ).

As it is evident from the list of taxa a total number of 48 species and subspecies which belong to 18 genera and 8 subfamilies have been established. The 15 taxa are new for the Albanian Buprestidae fauna. The new localities are established for the rest of the buprestids. With the collected new taxa the number of jewel beetles which are known from the Albanian territory is increased to 111 .

\section{Zoogeographical Results}

From the zoogeographical point of view all the established 111 buprestid taxa in Albania are arranged in 15 zoogeographical categories (Table 1).

Table 1. Zoogeographical characteristic of the Albanian buprestids.

\begin{tabular}{lc}
\hline \multicolumn{1}{c}{ TAXA } & ZOOGEOGRAPHICAL CATEGORIES \\
\hline Julodis ehrenbergi LAPORTE & Eastmediterranean \\
Ptosima flavoguttata (ILLIGER) & Southwestpalaearctic \\
Acmaeodera brevipes brevipes KIESENWETTER & Eastmediterranean \\
Acmaeodera pilosellae pilosellae (BONELLI) & Northmediterranean \\
Acmaeodera bipunctata bipunctata (OLIVIER) & Transmediterranean \\
Acmaeodera crinita crinita SPINOLA & Eastmediterranean \\
Acmaeodera ottonana (FRIVALDSZKY) & Eastmediterranean \\
Acmaeoderella jonica (OBENBERGER) & Eastmediterranean \\
Acmaeoderella adspersula adspersula & \\
(ILLIGER) & Transmediterranean
\end{tabular}


Acmaeoderella flavofasciata flavofasciata

(PILler et MitTerPacher)

Mediterranoeuropean

Acmaeoderella mimonti (BOIELDIEU)

Southwestpalaearctic

Chalcophora intermedia REY

Northmediterranean

Chalcophora mariana (LINNAEUs)

Eurosiberian

Chalcophora detrita (KLUG)

Eastmediterranean

Chalcophorella stigmatica (SCHÖNHERR)

Eastmediterranean

Chalcophorella fabricii (ROSSI)

Eastmediterranean

Capnodis tenebricosa (OLIVIER)

Southwestpalaearctic

Capnodis tenebrionis (LINNAEUS)

Southwestpalaearctic

Capnodis cariosa (PALlas)

Pontomediterranean

Capnodis porosa (KLUG)

Eastmediterranean

Cyphosoma euphraticum (LAPORTE \& GORY)

Eastmediterranean

Aurigena lugubris lugubris (FABRICIUS)

Pontomediterranean

Sphenoptera albanica OBENBERGER

Balkan endemic

Sphenoptera barbarica (GMELIN)

Transmediterranean

Sphenoptera lapidaria BRULLÉ

Eastmediterranean

Sphenoptera carceli (LAPORTE \& GORY)

Eastmediterranean

Sphenoptera aulacophora JAKOVLEV

Eastmediterranean

Sphenoptera rauca (FABRICIUS)

Southwestpalaearctic

Sphenoptera trebinjensis OBENBERGER

Balkan endemic

Sphenoptera laportei SAUNDERS

Mediterranoeuropean

Sphenoptera tappesi MARSEUL

Eastmediterranean

Dicerca moesta (FABRICIUS)

Eurosiberian

Dicerca aenea (LINNAEUS)

Transpalaearctic

Dicerca alni (FISCHER)

Westpalaearctic

Dicerca berolinensis (HERBST)

European

Scintillatrix mirifica MULSANT

European

Scintilatrix rutilans (FABRICIUS)

European

Eurythyrea quercus (HERBST)

European

Eurythyrea austriaca (LINNAEUS)

European

Eurythyrea aurata (PALLAS)

Pontomediterranean

Buprestis cupressi GERMAR

Eastmediterranean

Buprestis rustica LINNAEUS

Eurosiberian 
Buprestis haemorroidalis haemorroidalis HERBST

Westpalaearctic

Buprestis dalmatina MANNERHEIM

Eastmediterranean

Buprestis novemmaculata novemmaculata LINNAEUS

Transpalaearctic

Buprestis novemmaculata gravida ABEILLE DE PERRIN

Eastmediterranean

Buprestis splendens FABRICIUS

European

Melanophila acuminata (DE GEER)

Holarctic

Melanophila cuspidata (KLUG)

Transmediterranean

Phaenops cyanea (FABRICIUS)

Transpalaearctic

Anthaxia hungarica hungarica (SCOPOLI)

Mediterranoeuropean

Anthaxia diadema diadema (FISCHER)

Pontomediterranean

Anthaxia millefolii millefolii (FABRICIUS)

Pontomediterranean

Anthaxia umbellatarum umbellatarum (FABRICIUS)

Mediterranoeuropean

Anthaxia scutellaris scutellaris GENÉ

Westmediterranean

Anthaxia cichorii cichorii (OLIVIER)

Mediterranoeuropean

Anthaxia hypomelaena (ILLIGER)

Southwestpalaearctic

Anthaxia praeclara MANNERHEIM

Eastmediterranean

Anthaxia olympica KIESENWETTER

Pontomediterranean

Anthaxia similis SAUNDERS

European

Anthaxia istriana ROSENHAUER

Mediterranoeuropean

Anthaxia sepulchralis (FABRICIUS)

Westmediterranean

Anthaxia sturanyi OBENBERGER

Balkan endemic

Anthaxia helvetica STIERLIN

European

Anthaxia godeti LAPORTE et GORY

Southwestpalaearctic

Anthaxia quadripunctata (LINNAEUS)

Eurosiberian

Anthaxia funerula (ILLIGER)

Mediterranoeuropean

Anthaxia nitidula (LINNAEUS)

Westpalaearctic

Anthaxia discicollis LAPORTE \& GORY

Eastmediterranean

Anthaxia brevis brevis LAPORTE \& GORY

Pontomediterranean

Anthaxia fulgurans (SCHRANK)

European

Anthaxia podolica podolica MANNERHEIM

European

Anthaxia lucens KÜSTER

Eastmediterranean

Anthaxia deaurata (GMELIN)

Mediterranoeuropean 
Chrysobothris affinis (FABRICIUS)

Coroebus rubi (LINNAEUS)

Coroebus elatus (FABRICIUS)

Meliboeus amethystinus (OLIVIER)

Meliboeus graminis (PANZER)

Nalanda fulgidicollis (LUCAS)

Agrilus ater ater (LINNAEUS)

Agrilus biguttatus (FABRICIUS)

Agrilus convexicollis convexicollis REDTENBACHER

Agrilus derasofasciatus LACORDAIRE

Agrilus hastulifer RATZEBURG

Agrilus graminis LAPORTE et GORY

Agrilus dualis dualis ALEXEEV et BílÝ

Agrilus laticornis (ILLIGER)

Agrilus obscuricollis KIESENWETTER

Agrilus sulcicollis LACORDAIRE

Agrilus angustulus (ILLIGER)

Agrilus sinuatus (OLIVIER)

Agrilus pratensis pratensis RATZEBURG

Agrilus auricollis KIESENWETTER

Agrilus viridis (LINNAEUS)

Agrilus cuprescens MÉNÉTRIÉ

Agrilus lineola REDTENBACHER

Agrilus croaticus ABEILLE DE PERRIN

Agrilus marozzinii GOBBI

Agrilus viridicaerulans rubi SCHAEFER

Agrilus roscidus KIESENWETTER

Agrilus hyperici (CREUTZER)

Agrilus albaniae OBENBERGER

Paracylindromorphus subuliformis subuliformis (MANNERHEIM)

Aphanisticus pusillus (OLIVIER)

Aphanisticus emarginatus (OLIVIER)

Aphanisticus elongatus (VILLA)
Westpalaearctic

Southwestpalaearctic

Westpalaearctic

Westmediterranean

Mediterranoeuropean

Mediterranoeuropean

Westeurosiberian

Westpalaearctic

European

Mediterranoeuropean

Southwestpalaearctic

Southwestpalaearctic

Pontomediterranean

Westpalaearctic

European

European

Westpalaearctic

European

Westeurosiberian

European

Transpalaearctic

Transpalaearctic

European

Centraleuropean

Transmediterranean

Mediterranoeuropean

Southwestpalaearctic

European

Balkan endemic

Westpalaearctic

Mediterranoeuropean

Mediterranoeuropean

Mediterranoeuropean 
Trachys minutus (LINNAEUS)

Trachys scrobiculatus KIESENWETTER

Trachys troglodytes GYLLENHAL

Trachys coruscus PONZA
Eurosiberian

Mediterranoeuropean

European

Southwestpalaearctic

Following SAKALIAN's (1994) conception, the established 15 zoogeographical categories are combined in 7 zoogeographical complexes as follows: Holarctic complex with 1 species (Melanophila acuminata), Palaearctic complex (14 taxa) where Transpalaearctic (5) and Westpalaearctic (9) buprestids are included, Eurosiberian complex (7) represented by Eurosiberian (5) and Westeurosiberian (2) taxa, Southpalaearctic - including, in our case, 12 Southwestpalaearctic species, European complex (19) represented by 18 Transeuropean and 1 Centraleuropean taxa, Mediterranean complex (54) where belong taxa from 6 categories: Mediterranoeuropean (16), Transmediterranean (5), Northmediterranean (2), Westmediterranean (3), Pontomediterranean (8) and Eastmediterranean (20). The Balkan endemics are represented by 4 species. Up till now Sphenoptera albanica and Agrilus albaniae have been known only from the Albanian territory.

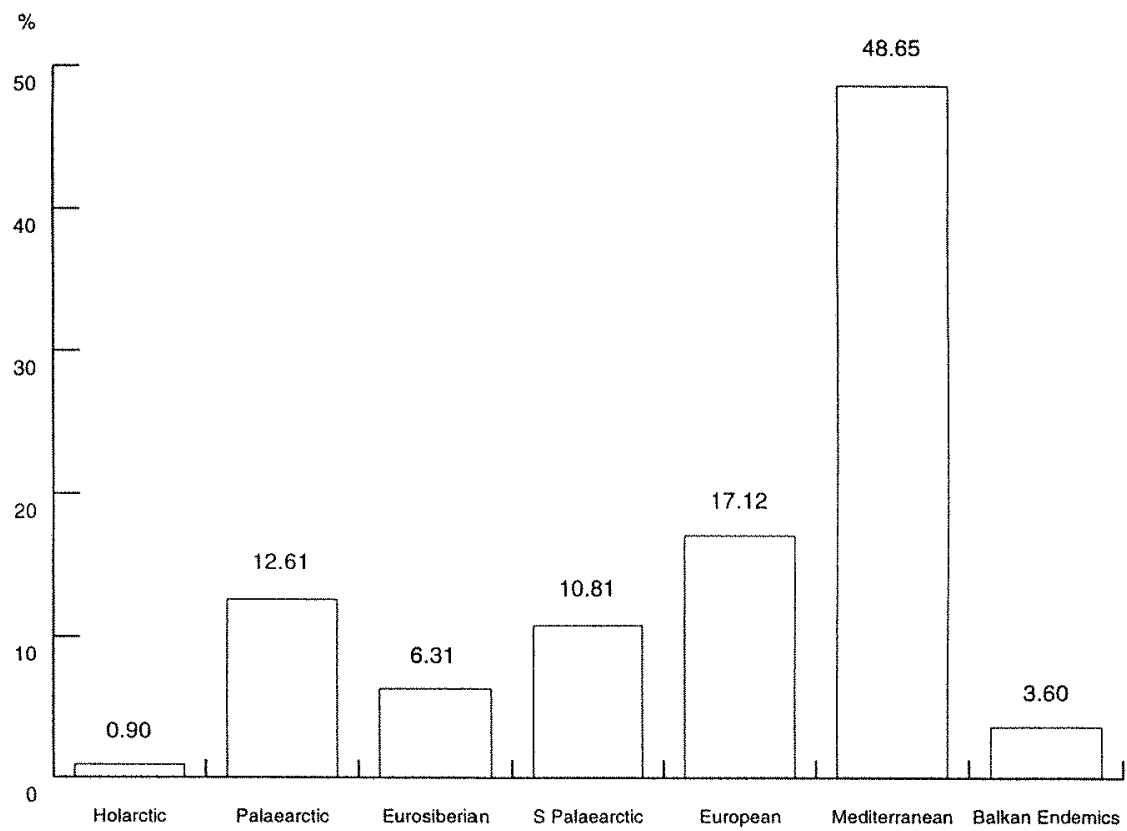

Fig. 2. Zoogeographical complexes in the study area.

The results of the zoogeographical analysis based on the presentation of these complexes in the studied area are shown in the Fig. 2.

The Mediterranean (in the broad sense) taxa are dominant in Albania as it is evident from the figure. More than $50 \%$ of the buprestids in the Mediterranean complex are Pontomediterranean and Eastmediterranean species and subspecies. 


\title{
Discussion
}

In our opinion the jewel beetles fauna of Albania is not studied enough. Nevertheless, the investigations by now, show that the great diversity of different natural habitat types in this country allows the distribution of many buprestid species there.

The great domination of Mediterranean taxa demonstrates the high level of the Mediterranean (especially Easmediterranean) fauna impact of buprestid community formation in the Albanian territory.

The location of relict Buprestis splendens and 4 Balkan endemics in Albania represent refugial peculiarity of some habitats in the country.

\section{References}

MÜHLE, H. 1980: Ergebnisse der Albanien-Expedition 1961 des Deutschen Entomologischen Institutes. 96. Beitrag (Coleoptera: Buprestidae). - Beiträge zur Entomologie 30 (2): 369-383.

MÜHLE, H. 1984: Ergebnisse der Albanien-Expedition 1961 des Deutschen Entomologischen Institutes. 97. Beitrag (Coleoptera: Buprestidae) (1. Nachtrag). - Beiträge zur Entomologie 34 (2): 425-426.

SAKALIAN, V. 1994: Studies on Buprestidae (Coleoptera) in the Sandanski-Petric and Goce Delcev valleys - Southwest Bulgaria. III. Zoogeographical characteristic. - Acta zoologica bulgarica 47: 35-42.

VOLKOVITSH, M. 1989: New and little known buprestid-beetles of the genus Acmaeoderella CoBos

(Coleoptera, Buprestidae) from the Eastern Mediterranea. - Proceedings of the Zoological Institute Leningrad 208: 43-63. [In Russian]

\author{
Author's address: \\ Dr. VLADIMIR SAKALIAN \\ Institute of Zoology \\ Bulgarian Academy of Sciences \\ Blvd. Tzar Osvoboditel 1 \\ BG -1000 Sofia \\ Bulgaria
}

\section{Besprechungen}

THIEDE, W.: Greifvögel und Eulen: Alle Arten Mitteleuropas erkennen und bestimmen. - München; Wien; Zürich: BLV Verlagsgesellschaft, 1999. - 95 S.: zahlr. Abb. - (BLV Naturführer). - ISBN 3-40515117-1. - 12.90 DM

Dieser Naturführer informiert über alle in Mitteleuropa häufig brütenden Greifvogel- und Eulenarten. Die gelungenen Farbfotos geben die faszinierenden Jäger naturgetreu wieder. Einer überblicksartigen Einführung folgen informative Vogelporträts, in denen die Merkmale exakt erklärt, Angaben zum Vorkommen der Arten und damit auch zum Zuchtverhalten und zum Winterquartier gemacht, die Nahrung und die Fortpflanzung beschrieben und Hinweise zur Gefährdung und zum Schutz der Tiere gegeben werden. 60 Graphiken zum Text zeigen Flugbilder oder geben Informationen zu Gefiedermerkmalen und zum Verhalten. In der Einführung geht es neben dem Überblick über die einzelnen Gruppen auch um die Gefährdung der Greifvögel und Eulen sowie um ihre Rolle im Leben des Menschen in verschiedenen Kulturkreisen.

Wie alle Bände des BLV Naturführers vermittelt auch dieser einen ersten Eindruck von der Vielfalt, vom Leben und vom Aussehen der behandelten Vogelgruppen. Wer Interesse an diesen Tieren gefunden hat, wird auf Adressen von Vereinen und Arbeitsgemeinschaften sowie auf weiterführende und vertiefende Literatur hingewiesen. 